Notfall Rettungsmed

https://doi.org/10.1007/s10049-021-00954-1

Angenommen: 29. September 2021

๑) Der/die Autor(en) 2021

\section{„Warum wird der Notruf 112 gewählt?" - Befragung zum Notrufverhalten der Berliner Bevölkerung}

\author{
Janosch Dahmen ${ }^{1,2,4}$. Paul Brettschneider ${ }^{1}$ Stefan Poloczek ${ }^{1,2}$. \\ Christopher Pommerenke ${ }^{1}$. Lisa Wollenhaupt ${ }^{1} \cdot$ Florian Breuer $^{1,3}$ \\ 'Berliner Feuerwehr, Berlin, Deutschland \\ ${ }^{2}$ Ärztliche Leitung Rettungsdienst Berlin, Berlin, Deutschland \\ ${ }^{3}$ Amt für Feuerschutz und Rettungswesen, Ärztliche Leitung Rettungsdienst Rheinisch-Bergischer Kreis, \\ Bergisch Gladbach, Deutschland \\ ${ }^{4}$ Fakultät für Gesundheit, Department Humanmedizin, Universität Witten/Herdecke, Witten, Deutschland
}

\section{Zusatzmaterial online}

Die Online-Version dieses Beitrags (https:// doi.org/10.1007/s10049-021-009541) enthält den zugrunde liegenden Fragebogen und Leitfaden für die Interviews.

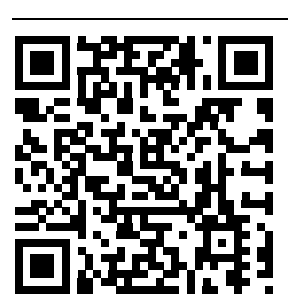

Hintergrund: Steigende Notrufzahlen und vermehrte Inanspruchnahme notfallmedizinischer Versorgungsstrukturen sind derzeit Gegenstand umfassender gesundheitspolitischer Debatten in Deutschland. Die vorliegende Arbeit untersucht in einer repräsentativen Befragung die Kenntnisse, das Verhalten und mögliche Ursachen in Zusammenhang mit dem Notruf 112 im Land Berlin.

Methode: In Zusammenarbeit mit Infratest dimap wurde eine Telefonumfrage nach dem Random-digit-dialing(RDD)-Verfahren mit einer repräsentativen Zufallsstichprobe von 1002 Personen der deutschsprachigen Berliner Wohnbevölkerung zu Kenntnissen und dem Umgang mit dem Notruf 112 durchgeführt. Eingeschlossen wurden Menschen im Land Berlin, die zum Befragungszeitraum mindestens 14 Jahre alt waren und über einen Festnetzanschluss verfügten. In 12 Fragen wurden Informationen zum Wissensstand über den Notruf 112, alternative Versorgungsstrukturen, das Nutzungsverhalten der Befragten und alternative Hilfsangebote einschließlich standardisierter Fallbeispiele abgefragt. Experteninterviews wurden ebenfalls durchgeführt.

Ergebnisse: $58 \%$ der Befragten gaben an, den Notruf 112 für medizinische Notfälle, $74 \%$ für Notfälle der Brandbekämpfung zu kennen. $91 \%$ der Befragten gaben an, dass sie den Notruf 112 nur bei lebensbedrohlichen medizinischen Situationen wählen würden. Befragte männlichen Geschlechts, ältere Menschen und Befragte mit einem niedrigeren Bildungsstand gaben häufiger an, den Notruf 112 auch bei nicht lebensbedrohlichen medizinischen Problemen in Anspruch zu nehmen. Alternativen zum Notruf kannten $56 \%$ der Befragten, wobei insbesondere die hausärztliche Versorgung kaum eine Rolle spielte. Experten sehen die fehlende Verfügbarkeit von Alternativen als ursächlich für die zunehmende Frequenz von Notrufen.

Schlussfolgerung: Fehlende suffiziente Alternativen in der Notfallversorgung könnten bei der vermehrten Inanspruchnahme des Notrufs eine Rolle spielen. Dennoch scheint die Berliner Bevölkerung durchaus mit der richtigen Nutzung des Notrufs vertraut zu sein. Die Gesundheitskompetenz von vulnerablen Gruppen sollte in Bezug auf das Wissen zum Notruf 112 gestärkt werden. Eine Bevölkerungskampagne könnte einen sinnvollen Ansatz darstellen. Weiterhin muss die Verfügbarkeit alternativer Versorgungsformen verbessert werden.

\section{Schlüsselwörter}

Notfallmedizin · Leitstelle · Prähospitale Versorgung · Qualitätsmanagement · Rettungsdienst 


\section{Hintergrund}

Seit 1973 werden bundeseinheitlich alle Notrufe der nichtpolizeilichen Gefahrenabwehr über die Notrufnummer 112 koordiniert. Der auf dem Gebiet der ehemaligen Deutschen Demokratischen Republik zwischenzeitlich zusätzlich etablierte Notruf 115 für medizinische Notfälle wurde inzwischen in eine allgemeine Behördenservicenummer umgewandelt und alle medizinischen Hilfeersuchen wurden einheitlich in den Notruf 112 integriert. Gemäß Beschluss der Europäischen Kommission und des Ministerrats der Europäischen Union (EU) wurde seit den 1990erJahren auch europaweit die einheitliche Notrufnummer 112 etabliert, die inzwischen auch viele Länder über die Europäische Union hinaus adaptiert haben, die Kenntnisse in Bezug auf den inzwischen etablierten einheitlichen Notruf 112 innerhalb der EU scheinen jedoch eher schlecht zu sein $[20,23]$.

Neben der Notrufnummer 112 und dem in Deutschland für die polizeiliche Gefahrenabwehr etablierten Notruf 110 existieren weitere Rufnummern für medizinische Hilfeersuchen, so etwa die bundeseinheitliche Rufnummer 116117 der kassenärztlichen Vereinigungen (KV) zur Vermittlung hausärztlicher Hilfe des ärztlichen Bereitschaftsdiensts bei nicht akut lebensbedrohenden Erkrankungen außerhalb der Öffnungszeiten der Arztpraxen. Darüber hinaus existieren beispielsweise für psychiatrische oder soziale Krisendienste oder auch für den Krankentransport oder Fragestellungen bei Vergiftungen (Giftnotruf) regional unterschiedliche Telefonnummern.

Sowohl in Deutschland als auch international ist in den vergangenen Jahren wiederholt über steigende Notruf- und Einsatzzahlen im Rettungsdienst berichtet worden. Mögliche Ursachen wurden dabei insbesondere im Bereich des demografischen Wandels, dem sich stark verändernden Gesundheitswesen und einer veränderten Selbsthilfefähigkeit der Bevölkerung gesehen [17]. Besonders im Bereich sozialer Brennpunkte, aber auch im Bereich organisierter Wohnformen, insbesondere bedingt durch eine zunehmende Anzahl von Menschen mit Pflegebedürftigkeit, ist von einer Zunahme von Hilfeersuchen auszugehen [1].

Gleichzeitig wurde in den vergangenen Jahren durch umfassende Kampagnen das Bewusstsein der Bevölkerung für Anzeichen einzelner Notfälle wie etwa Schlaganfälle („Jeder Schlaganfall ist ein Notfall“) oder Herzinfarkte („Herzinfarkt - Jede Minute zählt") gesteigert.

Die genauen Hintergründe steigender Einsatzzahlen und einer Zunahme von Hilfeersuchen über den Notruf 112 sind nach wie vor unklar. Eine mögliche Ursache könnte in mangelhaften Kenntnissen zum Notruf 112 bei der Bevölkerung liegen.

In der vorliegenden Arbeit werden im Ergebnis einer Bevölkerungsbefragung und von Experteninterviews Daten zur Motivation von Notrufen und zu Kenntnissen im Umgang mit dem Notruf 112 in Berlin dargestellt.

\section{Methodik}

Im vierten Quartal 2019 erfolgte in Zusammenarbeit mit der Firma Infratest dimap eine Telefonumfrage nach dem CATIVerfahren ("computer-assisted telephone interviewing"; [10]). Hierbei wurde mittels des Random-digit-dialing(RDD)-Verfahrens eine repräsentative Zufallsstichprobe von 1002 Personen der deutschsprachigen Berliner Wohnbevölkerung ausgewählt und mit einem standardisierten Fragebogen zu Kenntnissen und dem Umgang mit dem Notruf 112 befragt. Die Grundgesamtheit der Befragung bildete die deutschsprachige Bevölkerung ab einem Alter von 14 Jahren. Die Stichprobe spiegelt einen repräsentativen Querschnitt der Gesamtbevölkerung wider, indem die Verteilung von Merkmalen wie Altersgruppe oder regionaler Zugehörigkeit proportional zu den jeweiligen Anteilen der Gesamtbevölkerung ist. Eingeschlossen wurden Menschen im Land Berlin, die zum Befragungszeitraum mindestens 14 Jahre alt waren, in einem Privathaushalt lebten und über einen Telefonanschluss (Festnetz) verfügten. Die Verteilung der Teilnehmer an der Umfrage über die Berliner Bezirke war ausgeglichen.

Die Befragung erfolgte entsprechend dem ADM-Modell nach dem Gabler-Häder-Verfahren, welches zu verzerrungsfreien Stichproben ohne Klumpen führt [18].
In diesem Design verteilen sich die Merkmale der befragten Personen so wie in der Grundgesamtheit. Die Stichprobenziehung aus der Festnetzdatei erfolgte, da die Festnetznummern mit regionalen Zuordnungen versehen sind, sodass es möglich war, regional zu schichten. Der Erhebungszeitraum erstreckte sich über 18 Tage (25.09.2019-12.10.2019). Der Fragebogen (s. Zusatzmaterial online; siehe Box am Anfang des Artikels) enthielt insgesamt 12 Fragen. Die Fragen bezogen sich auf den Wissensstand über den Notruf 112 und alternative Versorgungsstrukturen, das Nutzungsverhalten der Befragten des Notrufs 112 und alternativer Hilfsangebote. Weiterhin wurden drei konkrete, standardisierte Fallbeispiele aufgeführt, zu denen Fragen in Bezug auf die richtige Hilfe gestellt wurden. Zum Teil waren Mehrfachantworten zulässig.

Bei den Befragten wurden die Altersgruppen 14-34, 35-49, 50-64 und 65+ unterschieden, beim Bildungsstand wurde unterschieden zwischen niedrigerer Bildung (Volks-/Hauptschule), mittlerer Bildung (mittlere Reife) und höherer Bildung (Abitur/Fachhochschulreife).

Die Berechnung der Konfidenzintervalle basiert auf der Formel für das Konfidenzintervall bei Zufallsstichproben mit großen Grundgesamtheiten $(K(p, n)=$ $1,96 \sqrt{(p(1-p) / n)})$, wobei in den Fällen, in denen die Formel nicht mehr angewendet werden konnte, kein Konfidenzintervall mehr angegeben wurde.

In einem zweiten Schritt wurden 5 Experten aus dem Bereich der medizinischen Notfallversorgung (national und international) unabhängig von der Bevölkerungsumfrage telefonisch anhand eines Leitfadens interviewt (s. Zusatzmaterial online; siehe Box am Anfang des Artikels). Die Experteninterviews zielten darauf ab, Hintergründe über die vermehrte Inanspruchnahme des Notrufs darzustellen. Hierbei handelte es sich um ärztliche Leiter von Rettungsdienst und Notaufnahmen, Vertreter der Ärztekammer, Patientenbeauftragte und Vertreter von Leitstellen. Die Interviews wurden aufgenommen und anschließend transkribiert. Es erfolgte eine qualitative Inhaltsanalyse nach Mayring [19]. 


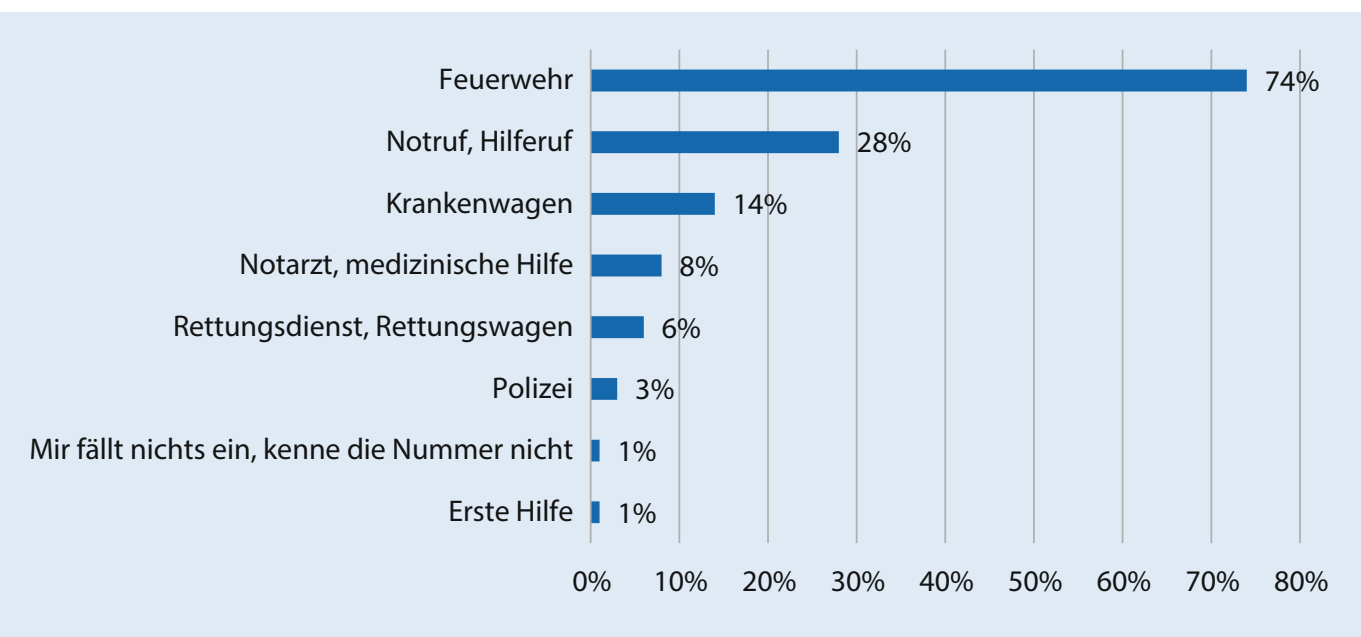

Abb. $1 \varangle$ Wissen der Bevölkerung über den Notruf 112. „Was fällt Ihnen spontan zur Telefonnummer 112 ein?" (Keine Antwortvorgabe, Mehrfachnennung möglich)

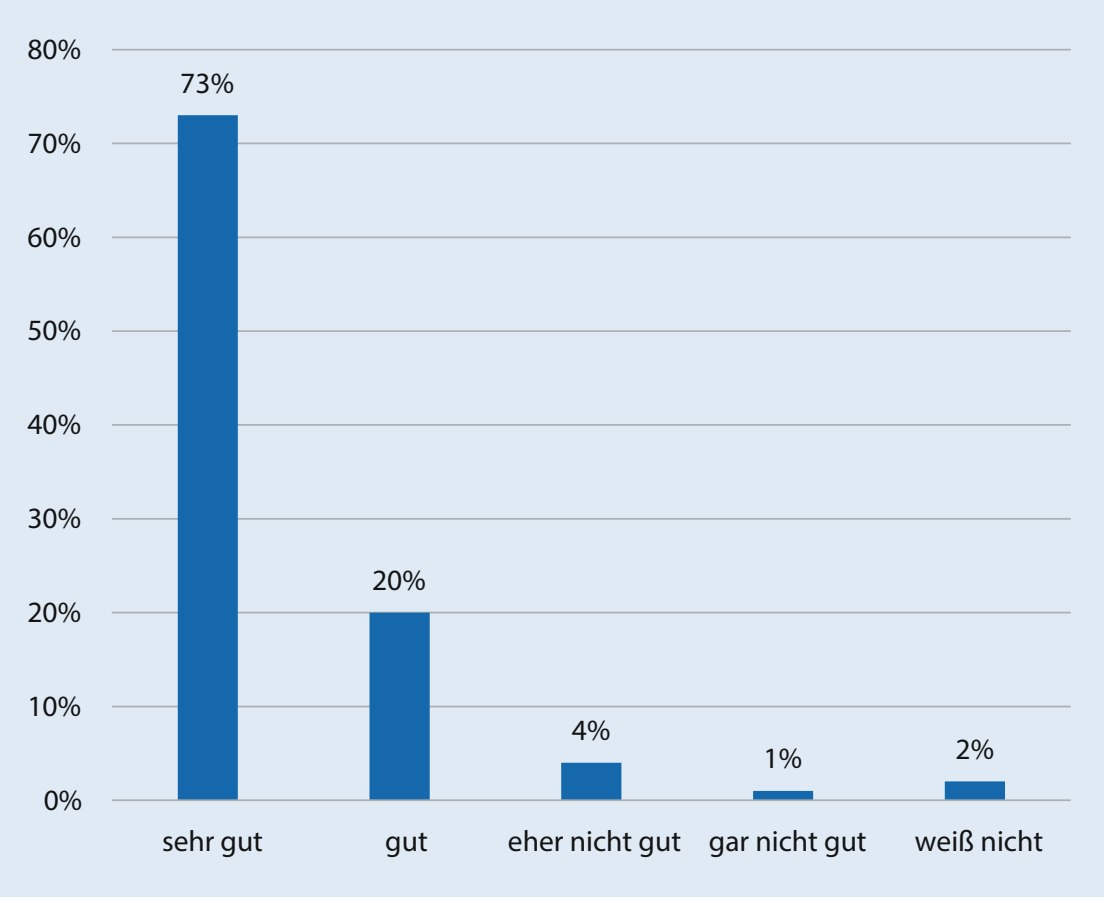

Abb. $2 \Delta$ Nutzung und Zufriedenheit bei denjenigen, die schon mal die 112 gewählt haben. „Wie wurde Ihnen da geholfen? Würden Sie sagen ...?"

\section{Ergebnisse}

Unter den 1002 Befragten befanden sich 489 (49\%) Männer und 513 (51\%) Frauen. Das Durchschnittsalter der Befragten betrug 47,3 Jahre. $11 \%$ der Befragten gaben als höchsten Schulabschluss einen Hauptoder Volksschulabschluss an, $40 \%$ die mittlere Reife, $46 \%$ die Allgemeine oder die Fachhochschulreife. $3 \%$ der Befragten gaben an, sich zum Befragungszeitraum noch in der Schulausbildung zu befinden.

$33 \%$ der Befragten leben in einem 1-Personen-Haushalt, $66 \%$ in einem
2- oder Mehrpersonenhaushalt. $6 \%$ gaben an, in einem Mehrgenerationenhaushalt mit Partnerin/Partner, Kind/Kindern oder Großeltern zu leben. 15\% der Befragten gaben an, einen Migrationshintergrund zu haben (selbst zugewandert oder aber ein oder beide Elternteile zugewandert).

\section{Kenntnisse zum Notruf 112}

74\% (95\%-KI 71,2-76,8\%) aller Befragten assoziierten die Telefonnummer 112 mit der Feuerwehr. In $28 \%$ (95\%-KI 25,2-30,8\%) wurde die 112 mit Not-
ruf/Hilferuf in Verbindung gebracht. 14\% (95\%-KI 11,8-16,2\%) assoziierten den Notruf mit Krankenwagen, 8\% (95\%KI 6,1-9,9\%) mit Notarzt/medizinischer Hilfe, $6 \%$ (95\%-KI 4,6-7,4\%) mit Rettungsdienst/Rettungswagen und $1 \%$ mit Erster Hilfe, wobei hier keine Antwortvorgabe existierte und Mehrfachnennungen möglich waren (- Abb. 1). In der Altersgruppe $>65$ Jahre gaben $10 \%$ (95\%-KI 5,8-14,2\%) an, mit dem Notruf den Rettungsdienst/ Rettungswagen zu assoziieren. 4\% der Menschen mit Migrationshintergrund gaben an, die Nummer nicht zu kennen, von allen Befragten gab $1 \%$ an, die 112 nicht zu kennen.

Über ein Drittel der Befragten (39\%, 95\%-KI 36-42\%) hat schon mal den Notruf gewählt (44\% weiblich, $56 \%$ männlich). $73 \%$ (95\%-KI 68,5-77,5\%) der befragten Berliner, die bereits einmal den Notruf 112 gewählt hatten, gab an, dass ihnen mit ihrem Anliegen durch die Leitstelle sehr gut geholfen wurde, $20 \%$ (95\%KI 16,1-23,9\%) eher gut, $4 \%$ (95\%-KI 2,1-5,9\%) eher nicht gut und $1 \%$ gar nicht gut (- Abb. 2). Hierbei war festzustellen, dass desto höher der Bildungsstand ist, desto weniger der Notruf gewählt wird (Schulabschluss Volks-/Hauptschule 52\%; 95\%-KI 42,2-61,8\% "ja, schon gewählt" Abitur/Fachhochschulreife 34\%; 95\%-KI 30-38\%). Im Vergleich seltener wird der Notruf in Mehrgenerationenhaushalten gewählt (18\%).

Auf die Frage, „wann der Notruf 112 gewählt werden solle", gaben $91 \%$ (95\%KI 89,1-92,9\%) der Befragten an, dass sie den Notruf 112 nur bei einer lebensbe- 


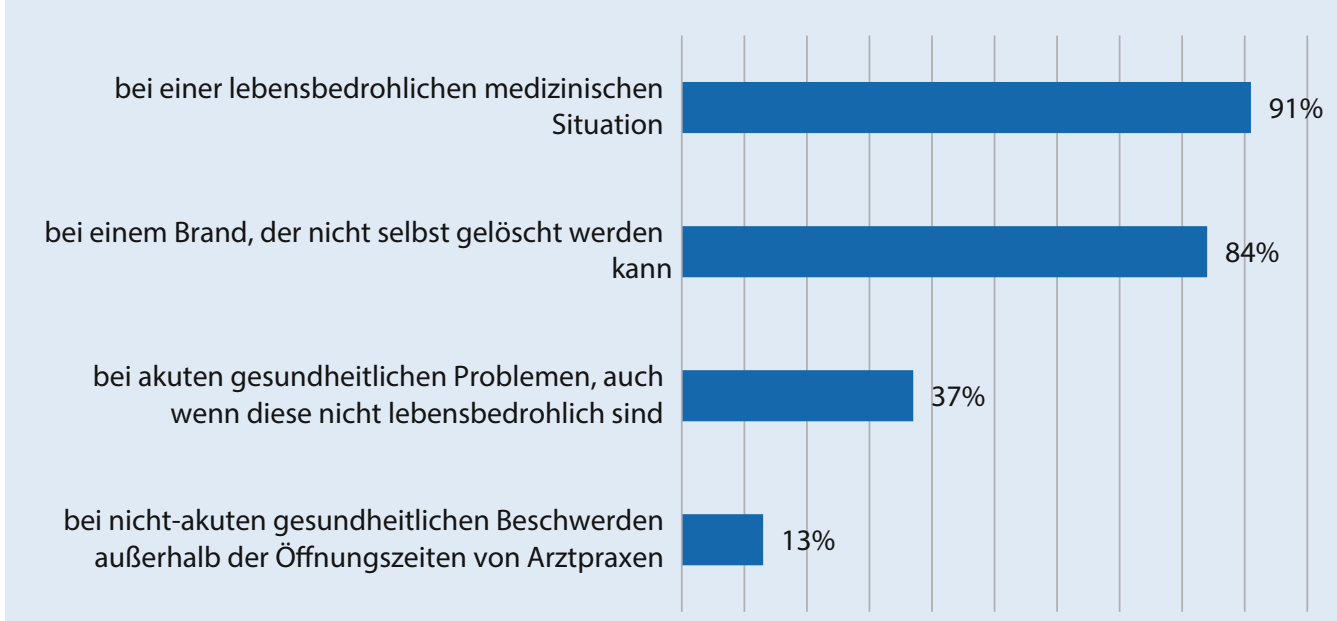

0\% $10 \%$ 20\% 30\% 40\% 50\% 60\% 70\% 80\% 90\% $100 \%$

Ich rufe die 112 nur bei Lebensgefahr an, wenn ich mir wirklich nicht mehr selbst helfen oder zu einem Arzt / Krankenhaus fahren kann.

Ich rufe die 112 nur im Ausnahmefall an, wenn ich mit meinem gesundheitlichen Problem wirklich nicht mehr anders weiterweiß.

Wenn mir ein gesundheitliches Problem eines Angehörigen über den Kopf wächst, bin ich froh, wenn die 112 und der Rettungsdienst mir helfen...

Ich zahle so wie andere Menschen Steuern, da darf man auch mal die 112 anrufen, wenn man ein akutes Problem hat

Der Hausarzt hat nachts und am Wochenende zu, Taxis sind teuer und im Krankenhaus muss man lange warten, die 112 und der Rettungsdienst...

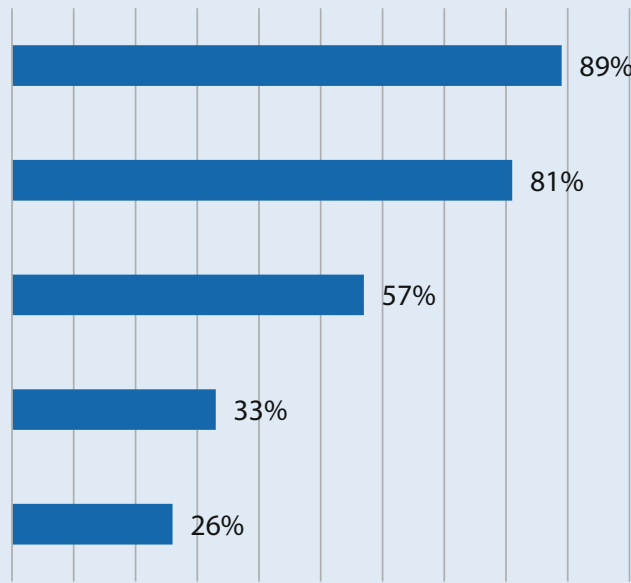

0\% 10\% 20\% 30\% 40\% 50\% 60\% 70\% 80\% 90\% 100\%
Abb. $3 \triangleleft$ Wissen über die Nutzung des Notrufs 112. „Was wissen Sie über die telefonische Notrufnummer ,112', wann sollte sie gewählt werden? Würden Sie sagen ..." (Mehrfachantworten möglich)
Abb. $4<$ Einstellung zur Nutzung des Notrufs 112. "Ich nenne Ihnen nun einige Aussagen zur Notrufnummer , $112^{\prime}$ und Sie sagen mir bitte, ob diese Aussage auf Sie zutrifft oder nicht. Wie ist das mit ..." (Mehrfachantworten möglich)

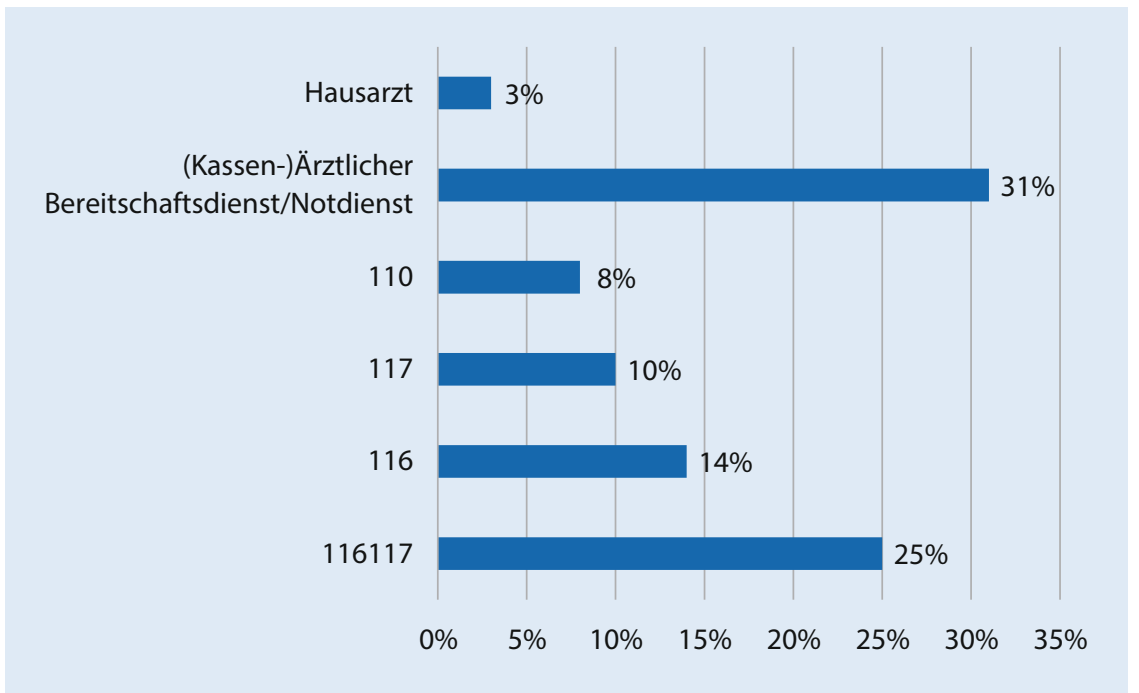

Abb. 5 ム Alternative Nummern zum Notruf. „Würden Sie mir bitte die alternativen Notrufnummern nennen, die Sie kennen?" (Ohne Antwortvorgaben, Mehrfachnennungen möglich) drohlichen medizinischen Situation wählen würden. $37 \%$ (95\%-KI 34-40\%) gaben an, dass sie den Notruf 112 bei akuten gesundheitlichen Problemen, auch wenn diese nicht lebensbedrohlich sind, wählen würden. $13 \%$ (95\%-KI 10,5-15,2\%) gaben an, den Notruf bei nichtakuten gesundheitlichen Beschwerden außerhalb der Öffnungszeiten von Arztpraxen zu wählen.

Weiterhin würden $84 \%$ (95\%-KI $81,5-86,5 \%)$ bei einem Brand, der nicht selbst gelöscht werden kann, den Notruf wählen (• Abb. 3).

$49 \%$ (95\%-KI 39,2-58,8\%) der Befragten in 1-Personen-Haushalten mit Kind(ern) gaben an, bei akuten gesundheitlichen Problemen, auch wenn diese nicht lebensbedrohlich sind, den Notruf zu wählen. 
Bitte stellen Sie sich folgende Situation vor: Es ist Samstagmittag. Bei Ihnen treten $\%$ plötzlich sehr starke Kopfschmerzen auf, die Sie so noch nie hatten. Was würden Sie tun? (Mehrfachnennungen möglich) Schmerztabletten einnehmen 60 Familie oder Bekannte um Rat fragen Am Montaq beim Hausarzt anrufen und einen Termin vereinbaren Den Ärztlichen Bereitschaftsdienst anrufen Zu einem Krankenhaus fahren Zu einer Notdienstpraxis des Ärztlichen Bereitschaftsdienstes fahren Am Montag beim Neurologen anrufen und einen Termin vereinbaren Die Notrufnummer 112 anrufen Weiß ich nicht Keine Angabe

\begin{tabular}{|c|c|}
\hline $\begin{array}{r}\text { Und was würden Sie in folgender Situation tun: Sie sind auf dem Weg zu einem } \\
\text { wichtigen Termin. An einer Bushaltestelle liegt eine offensichtlich verwahrloste } \\
\text { Person mit geschlossenen Augen am Boden. Die Person reagiert nicht auf Ansprache. } \\
\text { Was würden Sie tun? (Mehrfachnennungen möglich) }\end{array}$ & $\%$ \\
\hline \multirow{2}{*}{$\begin{array}{r}\text { Versuchen, wachzurütteln und zu fragen, ob dringend medizinische Hilfe benötigt } \\
\text { wird. Wenn ja, dann die Notrufnummer } 112 \text { anrufen } \\
\text { Andere Passanten ansprechen, um gemeinsam etwas zu tun und dann notfalls die } \\
112 \text { anrufen }\end{array}$} & 55 \\
\hline & 54 \\
\hline \multirow{8}{*}{$\begin{array}{r}\text { Erstmal vorsichtshalber die } 112 \text { anrufen und dann die Anweisungen befolgen } \\
\text { Weitergehen und die } 112 \text { anrufen, damit ich zumindest Bescheid gegeben habe } \\
\text { Nichts tun, vermutlich schläft die Person und ist in der Haltestelle ja vor Witterung } \\
\text { geschützt } \\
\text { Gar nichts tun, nicht ansprechen und auch wegen so etwas keinen Notruf wählen, } \\
\text { denn man hört ja so viele schlimme Geschichten } \\
\text { Nichts tun, ich bin für so etwas nicht geeignet und es kommen genug andere } \\
\text { Passanten vorbei } \\
\text { Weiß ich nicht } \\
\text { Keine Angabe }\end{array}$} & 48 \\
\hline & 13 \\
\hline & 4 \\
\hline & 1 \\
\hline & 1 \\
\hline & \\
\hline & 2 \\
\hline & 0 \\
\hline \multicolumn{2}{|l|}{$\begin{array}{l}\text { Und noch eine dritte Situation: Seit Wochen geht ein grippaler Infekt durch Ihren } \\
\text { Freundes- und Bekanntenkreis. Am Sonntagmorgen merken Sie, dass es auch Sie } \\
\text { erwischt hat, mit etwa } 38 \text { oder } 39 \text { Grad Fieber, Gliederschmerzen und Schnupfen. } \\
\text { Was würden Sie tun? (Mehrfachnennungen möglich) }\end{array}$} \\
\hline \multirow{4}{*}{$\begin{array}{r}\text { Am Montag zum Hausarzt gehen } \\
\text { Zur Notapotheke gehen und Medikamente besorgen } \\
\text { Familie und Bekannte um Rat fragen } \\
\text { Den Ärztlichen Bereitschaftsdienst anrufen }\end{array}$} & 63 \\
\hline & 39 \\
\hline & 32 \\
\hline & 18 \\
\hline \multirow{6}{*}{$\begin{array}{r}\text { Am Montag beim HNO-Arzt anrufen und einen Termin vereinbaren } \\
\text { Zu einer Notdienstpraxis des Ärztlichen Bereitschaftsdienstes fahren } \\
\text { Zu einem Krankenhaus fahren } \\
\text { Die Notrufnummer } 112 \text { anrufen } \\
\text { Weiß ich nicht } \\
\text { Keine Angabe }\end{array}$} & 16 \\
\hline & 12 \\
\hline & 10 \\
\hline & 6 \\
\hline & 2 \\
\hline & 2 \\
\hline
\end{tabular}

Abb. 6 \& Szenarien und Verhalten der befragten Bevölkerung

Weiterhin zeigte sich, dass Männer in $40 \%$ (95\%-KI 35,7-44,3\%) angaben, auch bei nicht lebensbedrohlichen Ereignissen den Notruf zu wählen, bei Frauen waren dies $35 \%$ (95\%-KI 30,8-39,2\%). Jüngere Menschen (14-34 Jahre) würden zu $18 \%$ (95\%-KI 13,5-22,5\%) bei nichtakuten gesundheitlichen Beschwerden außerhalb der Öffnungszeit von Arztpraxen anrufen.

Auf die Frage, ob die Aussage zutrifft, gaben $89 \%$ (95\%-KI 87,1-90,9\%) an, ,den Notruf 112 nur bei Lebensgefahr zu wählen", $81 \%$ (95\%-KI 78,5-83,5\%) gaben an, „im dringenden Ausnahmefall, wenn man mit den gesundheitlichen Problemen nicht mehr weiterweiß", den Notruf zu wählen (• Abb. 4).

$57 \%$ (95\%-KI 54-60\%) gaben an, „dass sie froh sind, wenn ihnen der Rettungsdienst hilft, wenn ihnen ein gesundheitliches Problem eines Angehörigen über den Kopf wächst und der Angehörige vorübergehend in ein Krankenhaus mitgenommen wird". Weitaus mehr befürworteten dieses in der Altersklasse über 65 Jahre $(70 \% ; 95 \%-K I$ 63,6-76,4\%) sowie bei niedrigerem Bildungsstand (64\%; 95\%-KI 54,4-73,6\%). Menschen mit Migrationshintergrund bejahten die Frage nur zu einem Anteil von $47 \%$ (95\%-KI 37,2-56,8\%).
$33 \%$ (95\%-KI 30-36\%) bejahten, „dass sie wie andere Menschen Steuern zahlen würden, daher dürfe man auch mal die 112 anrufen, wenn man ein akutes Problem hat". Bei der Altersgruppe über 65 Jahre bejahten dies $43 \%$ (95\%-KI 36,2-49,8\%). Einhergehend mit einem niedrigeren Bildungsstand wurde dies von $54 \%$ (95\%-KI 44,2-63,8\%) der Befragten bejaht.

$26 \%$ (95\%-KI 23,3-28,7\%) stimmten dahingehend zu, dass, "der Hausarzt am Wochenende zu hat, Taxen teuer seien und man im Krankenhaus lange warten müsse, der Rettungsdienst jedoch immer erreichbar sei, was auch schneller gehen würde". Menschen älter als 65 Jahre stimmten bei dieser Aussage zu 36\% (95\%-KI 29,4-42,6\%) zu. Von Befragten mit niedrigerem Bildungsstand wurde diese Aussage zu $43 \%$ (95\%-KI 33,2-52,8\%) bejaht.

Die Frage, ob bei einem dringenden, aber nicht lebensbedrohlichen Problem Alternativen zum Notruf 112 bekannt sind, bejahten 56\% (95\%-KI 53-59\%). Von denjenigen, die die Frage bejahten, wurden insbesondere 116117 (25\%), der ärztliche Bereitschaftsdienst (13\%) und der ärztliche Notdienst (12\%) genannt. $8 \%$ nannten die 110, nur $3 \%$ den Hausarzt (• Abb. 5).

\section{Notrufszenarien}

Im Rahmen der Befragung wurden weiterhin drei Szenarien mit Antwortvorgaben vorgestellt. Hierbei waren Mehrfachnennungen möglich (• Abb. 6).

Szenario 1. Es ist Samstagmittag. Bei Ihnen treten plötzlich sehr starke Kopfschmerzen auf, die Sie noch nie hatten. Was würden Sie tun? (Mehrfachnennungen möglich)

$60 \%$ (95\%-KI 57-63\%) gaben an, dann eine Schmerztablette einzunehmen, $43 \%$ (95\%-KI 39,9-46,1\%) würden die Familie oder Bekannte um Rat fragen. Den ärztlichen Bereitschaftsdienst würden 34\% (95\%-KI 31-37\%) anrufen. Nur $11 \%(95 \%-K I ~ 9,1-12,9 \%)$ würden die Notrufnummer 112 anrufen, wobei bei den Befragten älter als 65 Jahre 19\% (95\%-KI 13,5-24,5\%) den Notruf wählen würden. Jüngere Menschen (14-34 Jahre) gaben zu $67 \%$ (95\%-KI 61,8-72,2\%) an, auf eine Schmerztablette zurückzugreifen. 


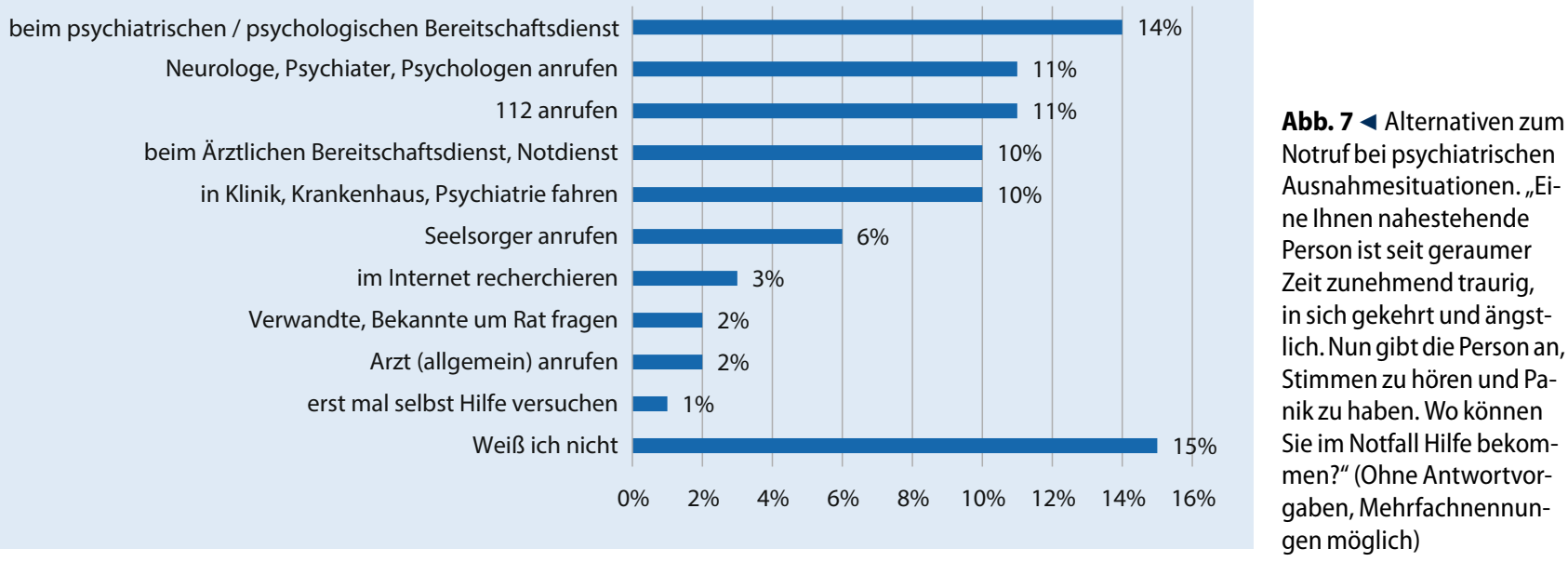

Szenario 2. Sie sind auf dem Weg zu einem wichtigen Termin. An einer Bushaltestelle liegt eine offensichtlich verwahrloste Person mit geschlossenen Augen am Boden. Die Person reagiert nicht auf Ansprache. Was würden Sie tun? (Mehrfachnennungen möglich)

$55 \%$ (95\%-KI 52-57,9\%) würden versuchen, die Person wachzurütteln, und fragen, ob dringend medizinische Hilfe erforderlich ist und erst dann den Notruf wählen. $13 \%$ (95\%-KI 10,8-15,2\%) würden weitergehen und die 112 anrufen, um zumindest Bescheid zu geben. Nur $1 \%$ hat angegeben, gar nichts zu tun. Auffallend ist, dass bei Personen mit niedrigerem Bildungsgrad $5 \%$ der Befragten gar nichts tun würden.

Szenario 3. Seit Wochen geht ein grippaler Infekt durch Ihren Freundes- und Bekanntenkreis. Am Sonntagmorgen merken Sie, dass es auch Sie erwischt hat, mit etwa 38-39 Grad Fieber, Gliederschmerzen und Schnupfen. Was würden Sie tun? (Mehrfachnennungen möglich)

$63 \%$ (95\%-Kl 60-66\%) gaben an, am Montag den Hausarzt aufzusuchen, $39 \%$ (95\%-KI 36-42\%) würden eine Notapotheke aufsuchen und 32\% (95\%-KI 29,2-34,8\%) die Familie oder Bekannte um Rat fragen. 18\% (95\%-KI 15,5-20,5\%) würden den ärztlichen Bereitschaftsdienst hinzuziehen, nur $6 \%$ (95\%-KI 4,6-7,4\%) gaben an, die Notrufnummer 112 zu wählen. Ältere über 65 Jahre gaben in $12 \%$ (95\%-KI 7,8-16,2\%) und Menschen mit geringerer Bildung gaben in $14 \%$ (95\%-
KI 7-21\%) an, die Notrufnummer 112 zu wählen.

\section{Akute psychiatrische Erkrankung}

Bei einer akuten psychiatrischen Erkrankung einer nahestehenden Person ist auffallend, dass $15 \%$ (95\%-KI 12,8-17,2\%) angegeben haben, nicht zu wissen, woher sie Hilfe bekommen ( $\bullet$ Abb. 7). Bei den 35 - bis 49-Jährigen waren dies sogar $20 \%$ (95\%-KI 14,5-25,5\%) und bei Menschen in 1-Personen-Haushalten $20 \%(95 \%-\mathrm{KI}$ 15,5-24,5\%). $11 \%$ (95\%-KI 9,1-12,9\%) würden die 112 anrufen, 14\% (95\%-KI $11,8-16,2 \%)$ würden sich an den psychiatrischen Bereitschaftsdienst oder den Krisendienst wenden.

\section{Hilfsbedürftigkeit im privaten Umfeld}

Auf die Frage, ob es im eigenen Familienoder Bekanntenkreis Menschen gibt, die Schwierigkeiten haben, sich in unserem Gesundheitssystem zurechtzufinden, zum Beispiel nicht wissen, an wen sie sich bei gesundheitlichen Problemen wenden können, antworteten 19\% (95\%KI 17,5-22,5\%) mit Ja und 79\% (95\%-KI 17,5-22,5\%) mit Nein. In der Altersgruppe 50-64 glauben $24 \%$ (95\%-KI 18-30\%), dass es solche Personen in ihrem Umfeld gibt. Befragte mit Migrationshintergrund gaben in $23 \%(95 \%-\mathrm{Kl} 14,5-31,5 \%)$ an, dass es solche Personen in ihrem Familien- oder Bekanntenkreis gibt, bei den Befragten ohne Migrationshintergrund waren dies 18\% (95\%-KI 15,2-20,8\%).
Die Personengruppe, welche in Mehrgenerationenhaushalten lebt, glaubt zu $27 \%$ (95\%-KI 18-36\%), dass es diese Menschen in ihrem Umfeld gibt.

\section{Ergebnisse Experteninterviews}

Allen Interviews gemeinsam war, dass seitens der Experten eine deutliche Belastung des notfallmedizinischen Bereichs gesehen wird (ansteigende Notrufzahlen, mehr Fälle in Notaufnahmen). Der Grund hierfür wird allerdings weniger bei der Bevölkerung gesehen, sondern vielmehr in der Struktur des Gesundheitssystems. Hierbei spielen die schlechte Verfügbarkeit von Hausärzten, aber auch fehlende Alternativen zum Notruf 112 eine Rolle. Weiterhin wird allgemein ein zunehmendes Bewusstsein für Krankheiten, beispielsweise Schlaganfall oder Herzinfarkt, in der Gesellschaft gesehen, aber auch eine zunehmende Hilflosigkeit, beispielsweise aufgrund eines fehlenden sozialen Netzwerks. Der Notruf wird als einzige verlässliche 24/7 verfügbare Hilfe für die Gesellschaft gesehen. Um diesen zu entlasten, müssten zum einen kompetente, konkrete und unmittelbar verfügbare Alternativen zum Notruf geschaffen und kommuniziert werden, zum anderen muss das Vertrauen in die ambulante respektive hausärztliche Versorgung gestärkt werden.

\section{Diskussion}

Die Berliner Feuerwehr koordiniert in einer zentralen Leitstelle für das Land Berlin alle Hilfeersuchen, die über den Notruf 112 
eingehen. Sämtliche Anrufe in der Leitstelle werden unter Anwendung eines standardisierten Notrufabfrageprotokolls abgefragt. Im Ergebnis der standardisierten Notrufabfrage (SNAP) wird in Abhängigkeit des ermittelten Leitsymptoms oder der geschilderten Beschwerden einer von 4608 Dispatch-Codes generiert, an denen wiederum spezifische Reaktionen in Form der Alarm- und Ausrückeordnung unter Berücksichtigung der Dringlichkeit, aber auch der notwendigen Qualifikation oder Ausstattung der benötigten Einsatzmittel angebunden sind. Im Jahr 2019 wurden seitens der Berliner Feuerwehr 343.660 Einsätze in der Notfallrettung sowie 34.998 Einsätze im Notfalltransport durchgeführt [2]. In Berlin wird regelhaft innerhalb von $24 \mathrm{~h}$ bis zu 3000-mal der Notruf 112 gewählt. In den letzten Jahren sind die Alarmierungen und Einsätze im Land Berlin stetig angestiegen [4].

Die Ergebnisse der Bevölkerungsumfrage in Berlin zeigen, dass die Befragten grundsätzlich gut über den Notruf 112 und die dazugehörigen Aufgabenbereiche informiert sind. Personen männlichen Geschlechts, ältere Menschen und Personen mit niedrigerem Bildungsstand scheinen eher den Notruf 112 zu wählen, weiterhin scheint der Notruf gerade bei Menschen mit Migrationshintergrund oft nicht bekannt zu sein, sodass möglicherweise Aufklärung in dem Bereich notwendig ist.

Es ist davon auszugehen, dass sich gerade jüngere Menschen oft durch die Einbeziehung von Freunden und Bekannten zu helfen wissen, wobei mit zunehmendem Alter die fachliche Einschätzung von Ärzten immer wichtiger wird. Für ältere Menschen scheint mit zunehmendem Alter jedoch die Notrufnummer 112 immer mehr an Bedeutung zu gewinnen.

Es zeigt sich, dass mehr als die Hälfte der Befragten eine Alternative zum Notruf kennt. Hierbei fällt jedoch auf, dass gerade die hausärztliche Versorgung in dem Zusammenhang kaum eine Rolle zu spielen scheint. In einer vorangegangenen Telefonbefragung in der Westpfalz konnte im Jahr 2017 festgestellt werden, dass nur $2 \%$ die Telefonnummer des ärztlichen Bereitschaftsdiensts kennen. Weiterhin war es nur $20,9 \%$ der Befragten möglich, zwischen dem kassenärztlichen Dienst und dem Notarzt zu differenzieren. Als Notruf- nummer des Rettungsdiensts war die 112 nur bei 29,3\% der Befragten bekannt [14]. Diese Ergebnisse können mit der hiesigen Umfrage in der Form nicht bestätigt werden, wenn auch die Methodik der Fragestellungen unterschiedlich ist. Wissensdefizite und fehlende Alternativen zeigen sich allerdings insbesondere bei Hilfeersuchen aus dem psychiatrischen Spektrum. Dabei gilt, dass psychosoziale Fragestellungen im rettungsdienstlichen Einsatzspektrum zunehmen und somit der Möglichkeit der Anbindung an alternative Versorgungsformen eine besondere Bedeutung zukommt [5, 6]. Dies ist insbesondere dahingehend problematisch, dass psychosozial verursachte Einsätze vermutlich mitunter die häufigste Einsatzursache bei Anrufern darstellen, die Sozialhilfeempfänger sind [13]. In einer bundesweiten Umfrage wurde festgestellt, dass insbesondere die Dringlichkeit häufig nicht richtig eingeschätzt werden kann [21].

Die Gesundheitskompetenz scheint unter den verschiedenen Subgruppen der Bevölkerung in Abhängigkeit von Alter, Sozialstruktur und etwaigem Migrationshintergrund unterschiedlich zu sein. Dies deckt sich mit Ergebnissen aus verschiedenen Untersuchungen, wonach insbesondere vulnerable Gruppen eine verminderte Gesundheitskompetenz vorweisen [24]. Es konnte gezeigt werden, dass durch eine Notrufkampagne die Anruffrequenz gesenkt werden kann und die Nutzung von alternativen Versorgungseinrichtungenerhöht wurde [22].

Neben der anrufenden Bevölkerung auf der einen Seite spielt aber auch die Leitstelle als zentrale 24/7 verfügbare Einrichtung eine wesentliche Rolle.

Es wird davon ausgegangen, dass der Anteil an nicht akut lebensbedrohlichen Einsätzen im großstädtischen Bereich bei ca. $60 \%$ liegt [12]. Hier ist es - um Hilfeersuchen adäquat weiterleiten zu können erforderlich, dass die Leitstelle auch auf geeignete Schnittstellen zu alternativen Versorgungsstrukturen zugreifen kann. Gerade den Leitstellen kommt somit dahingehend eine besondere Bedeutung zu, dass Hilfeersuchen, die unter der 112 einlaufen, gegebenenfalls auch effektiv weitergeleitet werden können [20]. Da es der Bevölkerung zunehmend schwer fällt, die richtige Rufnummer nachzuvollziehen, könnte ei- ne Verzahnung insbesondere der Rufnummern 112 und 116117 zu einem gemeinsamen Notfall- und Hilfeleistungssystem einen sinnvollen Ansatz darstellen. Weiterhin scheint auch eine größere Differenzierung von Einsatzmitteln, die direkt über die Leitstelle unter anderem für psychosoziale Notfälle disponiert werden können, ein sinnvoller Ansatz zu sein [11]. So konnten in Berlin im Rahmen eines Modellversuchs zur Umsetzung von derartigen Mobile-integrated-healthcare- bzw. Integrated-emergency-health-care-Konzepten im April 2020 bei 341 Einsätzen der Berliner Notfallrettung gezeigt werden, dass $49 \%$ der durch einen "NotSan-Erkunder" versorgten Patienten anstelle einer Krankenhauseinweisung an alternative, besser geeignete Versorgungsstrukturen angebunden werden konnten [6].

Bei Aufklärungskampagnen und Bewerbung von alternativen Rufnummern muss jedoch auch beachtet werde, dass in Bezug auf die zunehmende Bedeutung der Einhaltung von Qualitätsindikatoren bei Tracerdiagnosen wie beispielsweise der Prähospitalzeit beim Schlaganfall sowie beim ST-Hebungs-Infarkt der Laienaufklärung sowie der Bewerbung der Notrufnummer 112 auch eine hohe Wichtigkeit zukommt [16].

Das Eckpunktepapier 2016 hat den Leitstellen dahingehend eine zentrale Bedeutung zugewiesen, dass hierüber die agierenden Systeme für die Versorgung aller medizinischen Hilfeersuchen (Rettungsdienst, kassenärztlicher Notdienst, Notaufnahmen) zu koordinieren und abzustimmen sind [9]. Hierbei sind bereits auf der Basis des Sachverständigenrats zur Begutachtung der Entwicklung im Gesundheitswesen 2018 sowie seitens des Bundesministeriums für Gesundheit Vorschläge gemacht worden, dass es womöglich sinnvoll ist, die Notrufnummer 112 mit der Rufnummer des kassenärztlichen Notdiensts zusammenzulegen bzw. in einer organisatorischen Einheit zu betreiben [15]. Es sollte zum einen die Notrufannahme mittels standardisierter Notrufabfrage in der Rettungsleitstelle erfolgen, weiterhin ist es entscheidend, dass eine medienbruchfreie Datenübermittlung zwischen den verschiedenen Versorgungssystemen gewährleistet ist. Hierzu gehören auch die Möglichkeit, 
Hilfeersuchen medienbruchfrei an ambulante Versorgungssysteme abzugeben, sowie eine Schnittstelle zu alternativen Versorgungsformen (Pflegedienste, Sozialdienste; [8]).

In den letzten Jahren zeigte sich jedoch auch, dass vermehrt straf- und zivilrechtliche Klagen gegenüber einzelnen Leitstellen erfolgen, sofern ein Patient einen Schaden davongetragen hat. Dies bringt mit sich, dass es aufseiten der Leitstellen notwendig ist, auch bei Anrufen durch den kassenärztlichen Notdienst zunächst standardisiert abzufragen, um zu einem objektiven Ergebnis zu kommen. Hierbei ist es erforderlich, dass auch seitens der Kassenärztlichen Vereinigung eine standardisierte medizinische Ersteinschätzung erfolgt, was aktuell bereits in der Umsetzung ist [3].

\section{Schlussfolgerungen}

Zusammengefasst ist aus den Ergebnissen nicht ableitbar, dass auf der Seite der Bevölkerung ein falsches Verständnis für die Nutzung des Notrufs 112 vorherrscht. Vielmehr scheint dieser in vielen Fällen mangels Alternativen gewählt zu werden.

Zweifelsohne kommt den Leitstellen somit zunehmend eine GatekeepingFunktion zu [7]. Es ist somit notwendig, weiterhin die hausärztliche Versorgung zu stärken und die ambulanten Versorgungsstrukturen in ein Netzwerk zu integrieren, das auch mit der Rettungsleitstelle in Verbindung steht. Ein höheres Bewusstsein für Krankheiten führt zwangsläufig auch zu früheren Alarmierungen des Rettungsdiensts. Dies ist sicherlich dahingehend positiv zu bewerten, dass somit bedrohliche Krankheiten früher diagnostiziert und behandelt werden können. Zweifelsohne führt auch der demografische Wandel mit einer zunehmenden Alterung der Gesellschaft zu vermehrten Notrufen. Somit ist es dringend erforderlich, eine größere Zahl zuverlässiger Alternativen für medizinische Hilfeersuchen im Gesundheitswesen zu schaffen. Diese müssen sich zwingend am konkreten Bedarf orientieren. Erst anschließend kann es erkennbar effektiv und nachhaltig sein, die Bevölkerung flächendeckend über alternative Versorgungsformen aufzuklären. Im Kern wird es auf den gemeinsamen Auftrag aller
Akteure ankommen, dass Gesundheitssystem jenseits bestehender Barrieren, insbesondere in der Notfallversorgung, durch eine umfassende Reform zu verbessern, bei der sinnvolle Alternativen zum Notruf 112 ein wichtiger Baustein sein sollten.

\section{Methodenkritik}

Es wurden lediglich diejenigen Haushalte berücksichtig, die über einen Festnetzanschluss verfügen. Weiterhin wurden ausschließlich deutschsprachige Menschen in die vorliegende Befragung einbezogen.

\section{Fazit für die Praxis}

- Der Umfrage zeigt, dass der Notruf 112 für medizinische Hilfeersuchen sowie andere Notrufe der nichtpolizeilichen Gefahrenabwehr insbesondere der Brandbekämpfung in der Bevölkerung bekannt ist. Menschen mit Migrationshintergrund kennen den Notruf 112 weniger als der Durchschnitt der Gesamtbevölkerung.

- In einzelnen Subgruppen der Befragung zeigten sich Anzeichen einer vermehrten Inanspruchnahme des Notrufs 112 (hohes Alter, männliches Geschlecht, niedriger Bildungsstand).

- In den durchgeführten Experteninterviews wurde das Fehlen von zuverlässigen Alternativen, die 24/7 schnell, unmittelbar und konkret in Ersatz des Notrufs 112 in Anspruch genommen werden können, als wesentliche Ursache für die vermehrte Inanspruchnahme des Notrufs 112 identifiziert.

- Eine Bevölkerungskampagne könnte helfen, bestimmte vulnerable Gruppen zu erreichen und die Gesundheitskompetenz zu verbessern.

\section{Korrespondenzadresse}

\section{Dr. Florian Breuer}

Berliner Feuerwehr

Voltairestr. 2, 10179 Berlin, Deutschland

Florian.Breuer@berliner-feuerwehr.de

Funding. Open Access funding enabled and organized by Projekt DEAL.

\section{Einhaltung ethischer Richtlinien}

Interessenkonflikt. J. Dahmen, P. Brettschneider, S. Poloczek, C. Pommerenke, L. Wollenhaupt und F. Breuer geben an, dass kein Interessenkonflikt besteht.
Für diesen Beitrag wurden von den Autoren keine Studien an Menschen oder Tieren durchgeführt. Für die aufgeführten Studien gelten die jeweils dort angegebenen ethischen Richtlinien.

Open Access. Dieser Artikel wird unter der Creative Commons Namensnennung 4.0 International Lizenz veröffentlicht, welche die Nutzung, Vervielfältigung, Bearbeitung, Verbreitung und Wiedergabe in jeglichem Medium und Format erlaubt, sofern Sie den/die ursprünglichen Autor(en) und die Quelle ordnungsgemäß nennen, einen Link zur Creative Commons Lizenz beifügen und angeben, ob Änderungen vorgenommen wurden.

Die in diesem Artikel enthaltenen Bilder und sonstiges Drittmaterial unterliegen ebenfalls der genannten Creative Commons Lizenz, sofern sich aus der Abbildungslegende nichts anderes ergibt. Sofern das betreffende Material nicht unter der genannten Creative Commons Lizenz steht und die betreffende Handlung nicht nach gesetzlichen Vorschriften erlaubt ist, ist für die oben aufgeführten Weiterverwendungen des Materials die Einwilligung des jeweiligen Rechteinhabers einzuholen.

Weitere Details zur Lizenz entnehmen Sie bitte der Lizenzinformation auf http://creativecommons.org/ licenses/by/4.0/deed.de.

\section{Literatur}

1. Arntz HR, Poloczek S (2012) Wann sollte man den Rettungsdienst nicht alarmieren? Notfall Rettungsmed 15:661-666

2. Berliner Feuerwehr (2020) Jahresbericht 2019. https://www.berliner-feuerwehr.de/fileadmin/ bfw/dokumente/Publikationen/Jahresberichte/ jahresbericht2019.pdf.Zugegriffen: 11. Sept. 2020

3. Breckner A, Roth C, Wensing M, Paulus J (2020) Quo vadis 116117? Bundesweiter Überblick über den Status Quo und aktuelle Veränderungen. Gesundheitswesen 82:324-327

4. Breuer F, Pommerenke C, Wollenhaupt L, Brettschneider P, Poloczek S (2020) Vorkommen von Frequent Usern und Frequent Callern in einem großstädtischen Rettungsdienst: Indikatoren eines unzureichenden Gesundheits- und Sozialsystems? Notfall Rettungsmed 23:122-131

5. BreuerF, PommerenkeC, WollenhauptL,PoloczekS (2020) Alarmierungen der Berliner Notfallrettung zu obdachlosen Frequent Usern. Notarzt 36:16-23

6. Breuer F, Pommerenke C, Ziemen B, Stiepak JK, Poloczek S, Dahmen J (2020) Einführung von NotSan-Erkundern im Rahmen der COVID-19 Pandemie in der Berliner Notfallrettung. Notfall Rettungsmed. https://doi.org/10.1007/s10049020-00786-5

7. Chwojka CC, Novosad H, Spielbichler S, Krammel M (2015) Leitstelle - Gatekeeping mit Qualitätsanspruch. Der vielschichtige Weg des Patienten in das Gesundheitssystem. Notfall Rettungsmed 18:581-587

8. Enneker-Forum-Falkenstein, Thinktank der ClausEnneker-Stiftung (2019) Antworten auf die aktuellen Fragen zur zukünftigen notfallmedizinischen Versorgung der Bevölkerung in Deutschland. Ergebnis der Expertentagung „Enneker Forum Falkenstein 2019". Notfall Rettungsmed 22:733-737

9. Fischer M, Kehrberger E, Marung $H$, Moecke $H$, Prückner S, Trentzsch H, Urban B, Fachexperten der Eckpunktepapier Konsensus-Gruppe (2016) Eckpunktepapier zur notfallmedizinischen Versor- 
gung der Bevölkerung in der Prähospitalphase und in der Klinik. Notfall Rettungsmed 19:387-395

10. Infratest Dimap (2020) Telefonerhebungen CATI: Computer-Assisted-Telephoning-Interview. https://www.infratest-dimap.de/ueber-uns/waswir-tun/methoden/telefonerhebungen/. Zugegriffen: 10.Sept. 2020

11. Kristensen MS, Ersboll AK, Ahlmark N, Thomsen T (2018) The Sociolance: amobile clinic requested through emergency medical dispatch center serving socially vulnerable and homeless people in the capital city of Denmark. Rev Epidemiol Sante Publique 66(5):S371. https://doi.org/10.1016/j. respe.2018.05.363

12. Lechleuthner A, Wesolowski M, Brandt S (2019) Gestuftes Versorgungssystem im Kölner Rettungsdienst. Ein neuer Ansatz zur Bewältigung steigender Einsatzzahlen auf Basis einer neuen $\mathrm{Pa}$ tientenklassifizierungs- und Versorgunggstruktur. Notfall Rettungsmed 22:598-607

13. Luiz T, Huber T, Schieth B, Madler C (2000) Der psychosoziale Notfall. Notfall Rettungsmed 11:547-551

14. Luiz T, Dittrich S, Pollach G, Madler C (2017) Kenntnisstand der Bevölkerung über Leitsymptome kardiovaskulärer Notfälle und Zuständigkeit und Erreichbarkeit von Notrufeinrichtungen. Ergebnisse der KZEN-Studie in der Westpfalz. Anaesthesist 66:840-849

15. Sachverständigenrat zur Begutachtung der Entwicklung im Gesundheitswesen (2018) Sektorenübergreifende Ausgestaltung der Notfallversorgung. In: Bedarfsgerechte Steuerung der Gesundheitsversorgung, S547-597

16. Scherer G, Luiz T (2011) Eckpunktepapier zur notfallmedizinischen Versorgung der Bevölkerung in der Prä- und Hospitalphase. Wie ist es praktisch umsetzbar? Anaesthesist 60:751-758

17. Sieber F, Kotulla R, Urban B, Groß S, Prückner $S$ (2020) Entwicklung der Frequenz und des Spektrums von Rettungsdiensteinsätzen in Deutschland. Notfall Rettungsmed 23:490-496

18. Gabler S, Häder S (1998) Ein neues Stichprobendesign für telefonische Umfragen in Deutschland. In: Gabler S, Häder S, Hoffmeyer-Zlotnik JHP (Hrsg) Telefonstichproben in Deutschland. Westdt. Verlag, Opladen, S69-88

19. Mayring P (2015) Qualitative Inhaltsanalyse. Grundlagen und Techniken. Beltz Pädagogik. Beltz, Weinheim

20. Europäische Kommission (2020) Bericht der Kommission an das europäische Parlament und den Rat über die Wirksamkeit der Einführung der einheitlichen europäischen Notrufnummer 112. https:// digital-strategy.ec.europa.eu/en/library/2020report-effectiveness-implementation-europeanemergency-number-112. Zugegriffen: 26. Sept. 2021

21. Metelmann B, BrinkrolfP, Kliche $M$, Vollmer $M$, Hahnenkamp K, Metelmann C (2021) Rettungsdienst, Kassenärztlicher Notdienst oder Notaufnahme. Intensivmed Notfallmed. https://doi.org/10.1007/ s00063-021-00820-5

22. Borg K, Dumas D, Andrew E, Smith K, Walker T, Haworth M, Bragge P (2020) Ambulances are for emergencies; shifting behaviour through a research-informed behaviour change campaign. Health Res Policy Syst 18:9

23. Euopean Commission (2010) The European emergency number 112. Analytical report wave 3

24. Sorensen K, Pelikan JM, Röthlin F, Ganahl K, Slonska Z, Doyle G, Fullam J, Kondilis B, Agrafiotis D, Uiters $E$, Falcon $M$, Mensing $M$, Tchamov $K$, BrouckeS, Brand H (2015) Health literacy in Europe: comparative results of the European Healthliteracy survey (HLS-EU). Eur J Public Health 25:1053-1058
"Why do people call the German emergency number 112?": a survey on emergency call behavior in the Berlin population

Background: A rise in emergency calls and increasing utilization of the emergency medical services (EMS) are the subject of public health debates in Germany. This paper investigates, by means of a representative survey, the motivation and patterns behind emergency call use in the federal state of Berlin.

Method: In cooperation with Infratest dimap, a random-digit-dialing (RDD) phone survey was conducted, questioning 1002 randomly chosen individuals in Berlin's German-speaking population about their knowledge and usage patterns of the emergency call number 112. The survey included people of a minimum age of 14 years, whose homes are equipped with a landline. In all, 12 questions and example cases examined their knowledge about the emergency call number 112 and alternative care services, as well as their usage pattern of these. Additionally, EMS and public health experts were interviewed.

Results: A total of $58 \%$ of respondents stated being aware of the emergency call number 112 for medical emergencies and $74 \%$ for fire emergencies. Of the respondents, $91 \%$ stated that they would only call 112 in the case of life-threating medical emergencies. In all, $56 \%$ of respondents were able to name alternative care services. However, general practitioner care was underrepresented in the statements. Experts suggest that the lack of alternative care is the cause of the rising frequency in emergency calls.

Conclusion: The low availability of sufficient alternatives to EMS is a possible cause of increasing emergency calls. Nonetheless, the people of Berlin seem to be aware of the correct usage of the emergency call number. However, health literacy among vulnerable groups should be improved regarding knowledge about the emergency call number 112 . This could be achieved by a public campaign. Moreover, the availability of alternative care must be enhanced.

\section{Keywords}

Emergency medicine - Emergency medical dispatch center P Prehospital medical care · Quality management $\cdot$ Emergency medical service 